\title{
Autocrine factors sustain glioblastoma stem cell self-renewal
}

\author{
GUANGHUI LI ${ }^{1}$, ZHENGTANG CHEN $^{1}$, YI-DE HU ${ }^{1 *}$, HONG WEI $^{2 *}$, DEZHI LI $^{1}$, HUA JI $^{3}$ and DONG-LIN WANG ${ }^{1,4}$ \\ ${ }^{1}$ Institute for Cancer Research in People's Liberation Army, Xinqiao Hospital, Third Military Medical University, \\ Chongqing 400037; ${ }^{2}$ Department of Plastic Surgery, Southwest Hospital, Third Military Medical University, \\ Chongqing 400038; ${ }^{3}$ Department of Oncology, Kunming General Hospital of PLA, Kunming 650032, P.R. China
}

Received September 2, 2008; Accepted November 21, 2008

DOI: 10.3892/or_00000239

\begin{abstract}
Glioblastoma stem cells are able to reform original glioblastoma and express the neural stem cell marker CD133 and Nestin. They can self-renew and proliferate in tumor sphere medium containing EGF, bFGF and LIF that is known to be permissive for stem cell proliferation. In this study, we found that neurosphere-like colonies appeared after the human primary glioblastoma cells had been switched into pure DMEM/F12 medium. We investigated whether tumor spheres formed in pure DMEM/F12 medium possess the characteristics of glioblastoma stem cells. We identified that the tumor sphere cells were cancer stem cells of glioblastoma and they can self-renew and proliferate in pure DMEM/F12 medium. Glioblastoma cells can secrete several factors that result in autocrine motility signaling and stimulate glioma invasion. We hypothesized that an essential autocrine signal promotes the self-renewal and proliferation of human glioblastoma stem cells in pure DMEM/F12 medium. Then, expression of EGF and bFGF in glioblastoma stem cells were analyzed. Both the mRNA and protein of EGF and bFGF were detected in three human glioblastoma stem cells. Our findings suggest that autocrine of EGF and bFGF may sustain the self-renewal of glioblastoma stem cells.
\end{abstract}

\section{Introduction}

Cancer stem cells (CSCs) are widely thought to be able to initiate and reform the original cancer after CSCs were isolated and identified from more and more solid tumor and leukemia (1-5). They share many characteristics of embryonic stem cells and normal tissue stem cells. They possess the property of multipotential differentiation, unlimited proliferation and

Correspondence to: Dr Dong-Lin Wang, ${ }^{4}$ Present address: Department of Oncology, Tongji Hospital, Tongji University, Shanghai 200065, P.R. China

E-mail:wdl_tmmu@yahoo.cn

${ }^{*}$ Contributed equally

Key words: cancer stem cells, glioblastoma, autocrine, self-renewal, proliferation self-renewal $(6,7)$. The capacity of self-renewal plays a key role in retaining stem cell-like properties and maintaining CSC pool.

Brain tumor stem cells (BTSC) isolated from human glioblastoma (GBM) express the cell surface marker CD133, $(3,8,9)$ a neural stem cell marker. They are able to reform original glioblastoma and divide, amplify both undifferentiated and differentiated cancer cells after in vivo implantation. We have isolated BTSC from human primary glioblastoma tissue and identified its characteristics of CSCs (10). We found that a few glioblastoma cells were able to form neurosphere-like colonies while the primary human glioblastoma cells were switched into pure DMEM/F12 medium (without fetal bovine serum and exogenous growth factors). Then, the biological characteristics of this subpopulation of glioblastoma cells were analyzed. We demonstrated that the subpopulation of glioblastoma cells were BTSCs. The subpopulation also showed self-renewal in pure DMEM/F12 medium without fetal bovine serum (FBS) and exogenous growth factors. We show that there were expression of EGF and bFGF in BTSCs. Our findings suggest that autocrine of EGF and bFGF may sustain glioblastoma stem cell self-renewal.

\section{Materials and methods}

Culture of primary GBM cells and tumor sphere. Three human GBM samples were obtained from consenting patients, as approved by the Research Ethics Boards at The Xinqiao Hospital. Tumors were washed, acutely dissociated in DMEM/F12 medium without serum and subject to enzymatic dissociated. Tumor cells were then resuspended in DMEM/F12 medium containing $10 \%$ fetal bovine serum (FBS) as growth medium and plated at a density of $2 \times 10^{6}$ live cells per $75 \mathrm{~cm}^{2}$ flask. The cells attached and grew as a monolayer in flasks. The primary glioblastoma cells were harvested, dissociated and washed by pure DMEM/F12 medium three times. Then, all the three GBM cells were cultured in pure DMEM/F12 medium.

Subsphere forming assay. After primary spheres formed and reached 50-100 cells each in the culture, sphere cells were harvested, dissociated, washed by pure DMEM/F12 medium three times and plated in 96-well microwell plates for the subsphere-forming assay by limiting dilution, as described previously $(3,11)$. In brief, the cells in single-cell suspension were diluted and plated at 100 cells/well. Cells were fed with 
$0.2 \mathrm{ml}$ volumes of serum-free DMEM/F12 medium. Cultures were fed $0.025 \mathrm{ml}$ of fresh pure DMEM/F12 medium every 2 days. The wells were scored for sphere formation after 14 days.

Cell proliferation assay. The subsphere cells were harvested, dissociated into single cells, washed by pure DMEM/F12 medium three times and plated at 1000 cells/well into a 96-well plate in $0.2 \mathrm{ml}$ volumes of pure DMEM/F12 medium. Half of the medium in every well was changed every 2 days. Cell proliferation assays were performed on days $1,3,5,7$ and 9 postplating using the Dojindo WST-8 [2-(2-methoxy-4nitrophenyl)-5-(2,4-disulfophenyl)-2H-tetrazolium, monosodium salt]-based cell counting kit-8 (Dojindo Biotechnology, Japan). Quantification of viable cells through reading of UV absorption spectrums at $450 \mathrm{~nm}$ was performed on a microplate reader.

Immunocytochemical staining of GBM sphere cells. For immunostaining of undifferentiated tumor spheres, spheres were plated onto poly-L-lysine coated glass coverslips in pure DMEM/F12 medium for $4 \mathrm{~h}$. Cells were then fixed with $4 \%$ paraformaldehyde and stained with antibodies against CD133 (goat polyclonal IgG; Santa Cruz Biotechnology), Nestin (mouse monoclonal IgG; Santa Cruz Biotechnology), glial fibrillary acidic protein (GFAP, mouse monoclonal IgG; Santa Cruz Biotechnology), myelin basic protein (MBP, rabbit polyclonal IgG; Santa Cruz Biotechnology), mitogen-activated protein 2 (MAP2, mouse monoclonal IgG; Santa Cruz Biotechnology). Appropriate SP Cytostain-Plus kits (Zhongshan Biotechnology) were used. Color visualization was performed using 3-3'-diaminobenzidine as the chromagen substrate (Zhongshan Biotechnology).

The glioblastoma sphere cells were dissociated into single cells. CD133-2-phycoerythrin (10 $\mu 1)$ (fluorochromeconjugated mouse monoclonal $\operatorname{IgG1}$; Miltenyi Biotec) was added for $30 \mathrm{~min}$ and the percentage of $\mathrm{CD} 133^{+}$cells were counted by flow cytometry. The experiment was repeated three times.

Implantation into nude mice. Five Balb/C nude mice (6 weeks old; The Experimental Animal Laboratories, Shanghai, P.R. China) were anesthetized with i.p. ketamine and xylazine and then were treated with VP-16 (etoposide), $30 \mathrm{mg} / \mathrm{kg}$, diluted in serum-free HBSS, via an i.p. injection. Seven days later, the mice were anesthetized as described above and the regions of the upper extremity were implanted subcutaneously with both isolated GBM sphere cells (right, 10000 cells per mouse) and primary GBM cells (left, 1000000 cells per mouse) in $0.2 \mathrm{ml} 0.01 \mathrm{M} \mathrm{PBS}$. Tumor growth was monitored weekly. The mice were sacrificed after 6 weeks of the implantion. The same experiment was repeated once with identical situations. All the animal experiments were in strict accordance with the Institutional Animal Care guidelines.

Immunohistochemistry on tumor sections. Formalin-fixed, paraffin-embedded tissue sections were mounted on positivecharged microscope slides. Tissue sections were then baked overnight at $60^{\circ} \mathrm{C}$ and treated with epitope retrieval techniques and blocked for endogenous peroxidase and biotin before the application of the primary antibody. Incubation of antihuman GFAP at a 1:100 dilution was performed overnight at $4^{\circ} \mathrm{C}$. Subsequent immunodetection was performed using SP Histostain-Plus kits (Zhongshan Biotechnology). Color visualization was performed using 3-3'-diaminobenzidine as the chromagen substrate (Zhongshan Biotechnology).

RNA extraction and RT-PCR. Total RNA of glioblastoma subsphere cells was extracted by Tripure Reagent (Roche, USA). Retrotranscription was carried out with RNA for each sample using RT M-MLV (Toyobo, Japan) in the presence of random primers. The PCR was carried out in a $20 \mu 1$ reaction mixture that contained $1 \mu \mathrm{l}$ cDNA as template, specific oligonucleotide primer pairs, and Accuzyme (Toyobo). EGF (forward GGATAGCCAACAAACACACT, reverse GGCACGTGCAGTAATAGGAT): 36 cycles of $30 \mathrm{sec}$ at $94^{\circ} \mathrm{C}, 30 \mathrm{sec}$ at $60^{\circ} \mathrm{C}$ and $90 \mathrm{sec}$ at $72^{\circ} \mathrm{C}$; bFGF (forward CCTGGGGAGAAAGCTAT, reverse GCTTCACGGGTAA CAG): 36 cycles of $30 \mathrm{sec}$ at $94^{\circ} \mathrm{C}, 30 \mathrm{sec}$ at $58^{\circ} \mathrm{C}$ and $90 \mathrm{sec}$ at $72^{\circ} \mathrm{C}$. Sense and antisense primers were synthesised according to the sequences extracted from GenBank. The amplified products were identified by agarose gel electrophoresis and ethidium bromide staining.

Immunoblotting. Cell extracts were prepared with RIPA kit (KeyGen Tec, Nanjing, P.R. China) and resolved on a $10 \%$ SDS-PAGE and blotted onto a membrane. After blocking at $37^{\circ} \mathrm{C}$ in $5 \%$ milk in PBS containing $0.1 \%$ Tween-20 for $2 \mathrm{~h}$, membranes were incubated overnight with antibodies against EGF, bFGF or ß-actin proteins (Santa Cruz Biotechnology). After extensive washing, immunocomplexes were detected with horseradish peroxidase-conjugated appropriate secondary antibodies followed by standard chemiluminescent substrate (Pierce Biotechnology).

\section{Results}

Primary GBM form neurosphere-like colonies in pure $D M E M / F 12$ medium. Studies on BTSCs have showed that BTSCs is able to proliferate and form neurosphere-like colonies cultured in serum-free tumor sphere medium (TSM) containing EGF, bFGF and $\operatorname{LIF}(3,8,12)$. When primary GBM cells were switched into pure DMEM/F12 medium, neurosphere-like colonies appeared in all three GBM and reached $\sim 50$ cells each sphere in 2 weeks (Fig. 1A). It was smaller than the tumor spheres cultured in TSM. The tumor spheres generated from 3 specimens remained nonaderent and exhibited immunoreactivity for CD133 and Nestin. A few cells within tumor spheres were also found to be positive for lineage markers MBP (for oligodendrocytes), but no GFAP (for astrocytes) and MAP2 (for neurons) staining was detected within spheres (Fig. 1B-F). The cells were stained with antibody conjugated PE against CD133. The frequency of $\mathrm{CD}_{133}{ }^{+}$cell population within the primary tumor spheres was determined by flow cytometric assays, yielding CD133+ cell frequency to $68.8 \%$ (Fig. 2).

GBM sphere cells exhibit potential for self-renewal and can proliferate in pure DMEM/F12 medium. Tumor spheres are defined as clonally derived nonadherent colonies of cells 
A tumor sphere

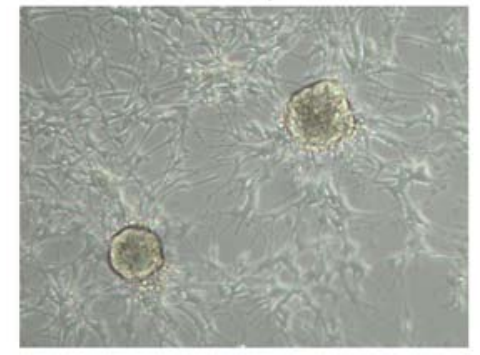

D

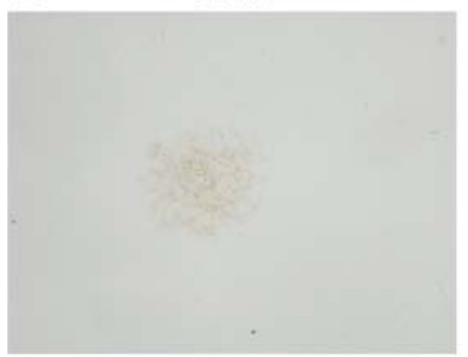

B

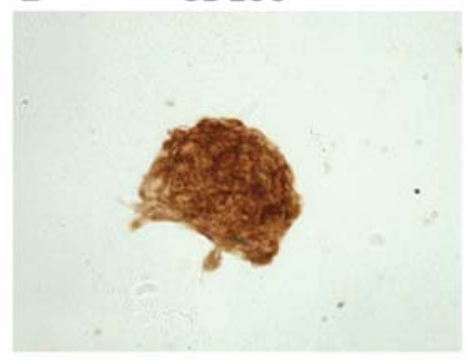

E

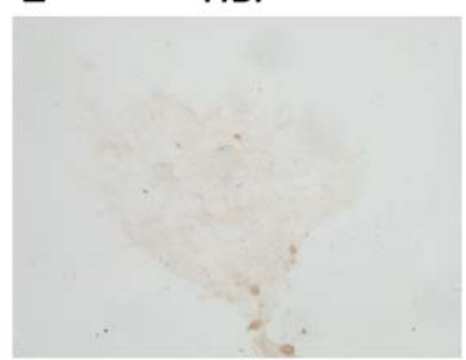

C

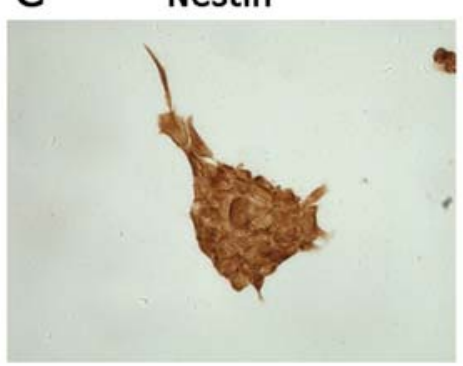

$\mathrm{F}$

MAP2

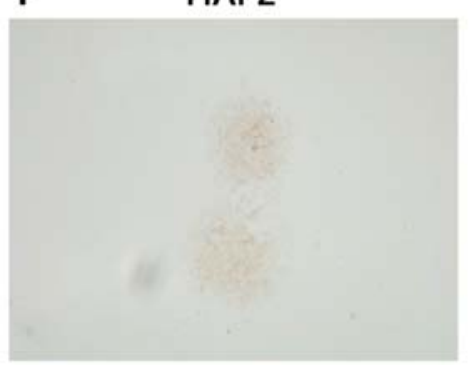

Figure 1. Neurosphere-like colonies appeared in GBM cells cultured in pure DMEM/F12 medium (A, magnification x100). Glioblastoma spheres were stained by NSC markers CD133 (B, magnification x400) and Nestin (C, magnification x400). Lineage markers for oligodendrocyte, MBP (E, magnification x400), were stained, but staining for the astrocyte marker, GFAP (D, magnification x400), and neuron, MAP2 (F, magnification x400), was not observed in the glioblastoma sphere.

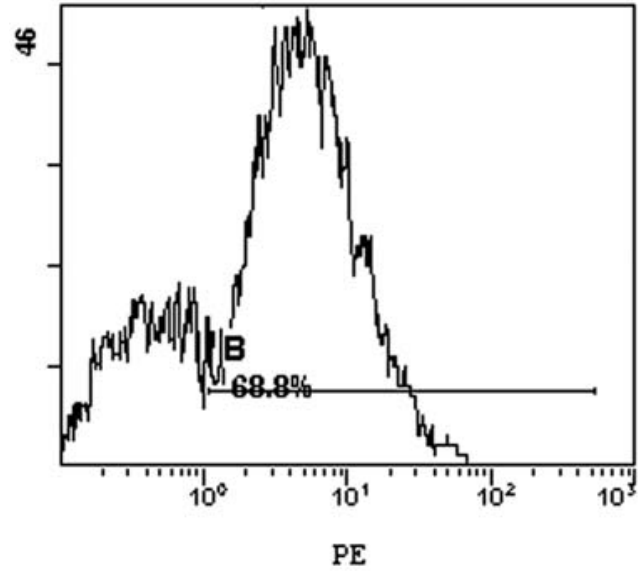

Figure 2. Flow cytometry histogram in representative glioblastoma sphere cells, with the first peak (left) representing cells negative for CD133phycoerythrin expression and the second peak (right, gate B) representing CD133-positive cells.

derived from a single tumor stem cell. We analyzed the ability of the primary GBM sphere cells to continue proliferating and forming secondary sphere with a sphere-forming assay. After disassociation and replating at $\sim 100$ cells/well, all of the dissociated primary tumor spheres demonstrated the capacity to form secondary tumor spheres, exhibiting ability to selfrenew in pure DMEM/F12 medium. It generated 5.04 \pm 2.39 secondary tumor spheres every 100 primary sphere cells. Morphology of secondary tumor spheres was identical to that of primary spheres and secondary tumor spheres retain expression of the neural stem cell markers CD133 and Nestin (Fig. 3). These data show that the CSCs of human glioblastoma had the capacity to self-renewal in pure DMEM/F12 medium.
The primary sphere cells were plated at 1000 cells/well cultured in pure DMEM/F12 medium and the number of viable cells was quantified on days 1,3,5,7 and 9 after plating by the WST- 8 colorimetric assay. It was demonstrated that the primary glioblastoma sphere cells were able to proliferate when they were cultured in pure DMEM/F12 medium. But its capacity of proliferation in pure DMEM/F12 medium was significantly lower than BTSCs cultured in TSM (Fig. 4). These data show that the primary glioblastoma spheres formed in pure DMEM/F12 medium had the capacity for self-renew and proliferation.

Glioblastoma spheres cultured in pure DMEM/F12 medium are capable of forming tumors in vivo. To determine whether the glioblastoma spheres are capable of tumor initiation in vivo, we implanted the isolated glioblastoma spheres cultured in pure DMEM/F12 medium into the upper extremity of nude mice subcutaneously. There was no tumor detected 6 weeks after the implantation of 1000,000 primary glioblastoma cells. Three mice developed tumor after 10,000 tumor sphere cells implantation in 5 mice. The tumor cells, which developed in mice after subcutaneous implantation of human glioblastoma spheres, stained positive for anti-GFAP antibody (Fig. 5). These results suggested that the glioblastoma spheres cultured in pure DMEM/F12 medium had the capacity to initiate tumor in vivo and possess characteristics of BTSC.

Glioblastoma sphere cells can autocrine EGF and bFGF. Astrocytes, oligodendrocytes and microglia can express EGF, hepatocyte growth factor (HGF), transforming growth factor- $\alpha$ (TGF- $\alpha$ ) and others (13-15). Glioblastoma cells also secrete several factors that result in autocrine motility signaling and stimulate glioma invasion (15-17). The 

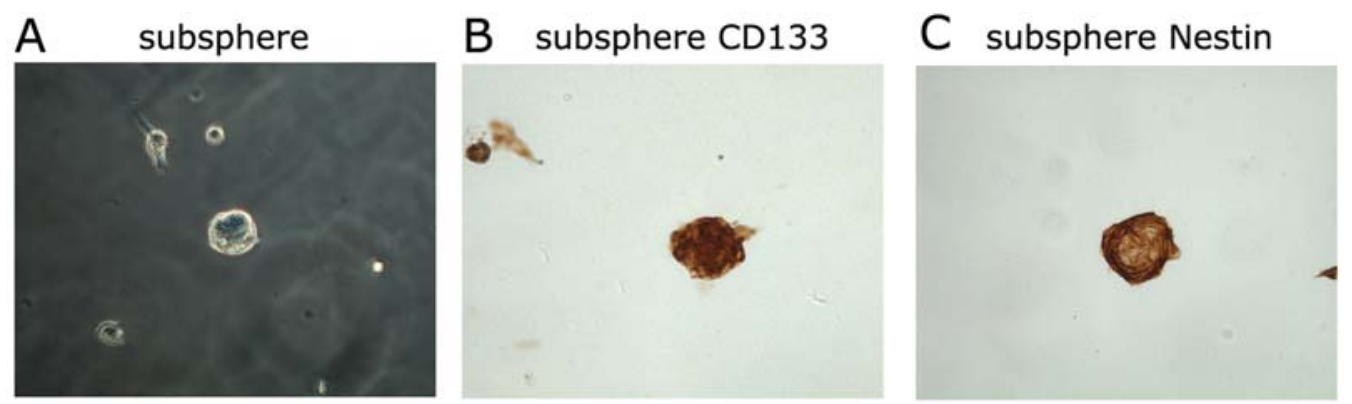

Figure 3. Tumor sphere cells exhibit self-renewal potential. Morphology of secondary tumor spheres (magnification x200) was identical to that of primary tumor spheres (A). Undifferentiated secondary tumor spheres (magnification x400) retain expression of neural stem cell markers CD133 (B) and Nestin (C).

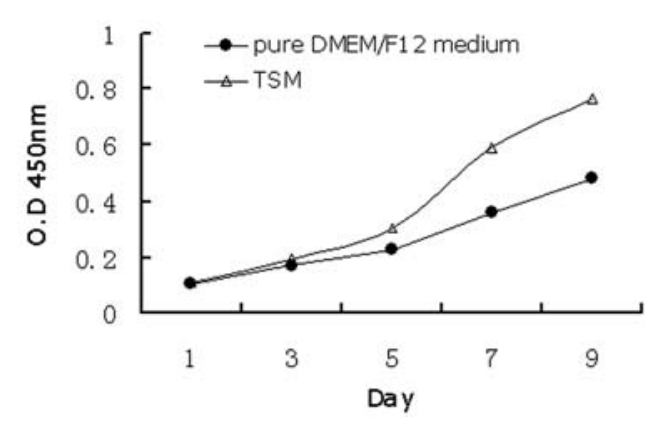

Figure 4. Tumor sphere cells exhibit proliferation cultured in pure DMEM/F12 medium. Tumor sphere cells cultured in pure DMEM/F12 medium showed a lower degree of cell proliferation than cells cultured in TSM. epidermal growth factor receptor (EGFR) gene is commonly amplified and rearranged in glioblastoma multiforme. Expression of wild-type EGFR ligands, such as transforming growth factor- $\alpha$ (TGF- $\alpha$ ) or heparin-binding EGF (HB-EGF), is also often increased in gliomas resulting in an autocrine loop that contributes to the growth autonomy of glioma cells $(15,16)$. Therefore, we hypothesized that an essential autocrine signal promotes the self-renewal and proliferation of human glioblastoma stem cells in pure DMEM/F12 medium. To better define the autocrine of glioblastoma stem cells, the total RNA and protein of glioblastoma subsphere cells were extracted. Expression of EGF and bFGF was assessed by RT-PCR and Western blot assay. As shown in Fig. 6 EGF and bFGF mRNA were amplified in all three glioblastoma subspheres. The protein of EGF and bFGF also were detected in three glioblastoma stem cells. These results are consistent with the above hypothesize on autocrine of glioblastoma stem cells.
A

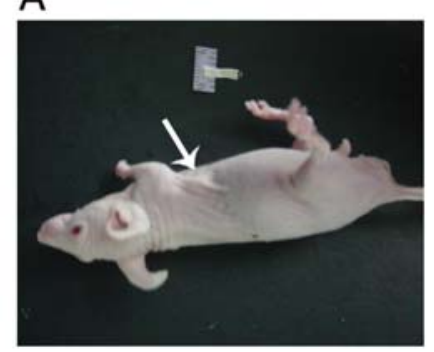

B

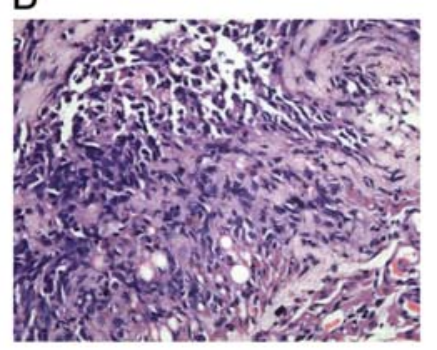

C

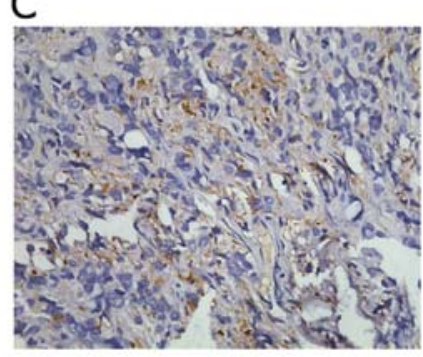

Figure 5. Evidence of in vivo tumor-forming ability of glioblastoma spheres. The isolated glioblastoma spheres were able to form tumors in nude mice after subcutaneous implantations (A), but the primary glioblastoma cells did not, even when implanted cell number reached 100 times that of the glioblastoma sphere cells. The tumors developed in the mice were stained by hematoxylin and eosin and could be stained by human-specific antibodies against GFAP.

A EGF

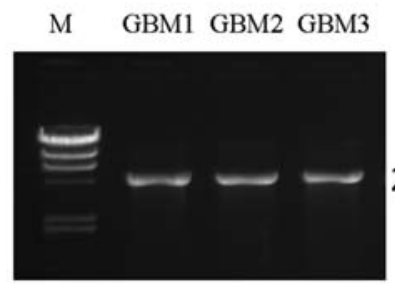

B bFGF M GBM1 GBM2 GBM3

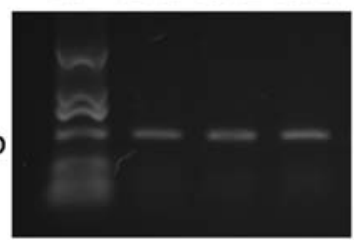

C

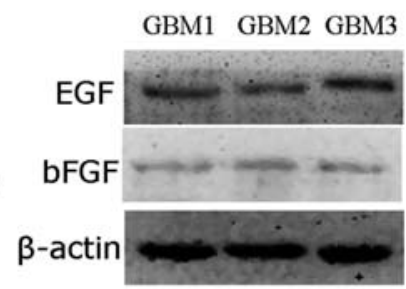

Figure 6. Semiquantitative RT-PCR and Western blot analysis of expression of EGF and bFGF in glioblastoma stem cells. Semiquantitative RT-PCR showed mRNA transcription levels of EGF (A) and bFGF (B) in three cases of glioblastoma stem cells. Western blot analysis confirmed levels of EGF and bFGF protein in three cases of glioblastoma stem cells (C). 


\section{Discussion}

The concept of CSC arose from the striking similarities between the self-renewal mechanisms of stem cell and a small fraction of cancer cells in tumors (7). One key determinant of stem cells and CSCs is the capacity for self-renewing and extensive proliferation $(18,19)$. BTSCs were isolated with a remarkable potential for self-renewal and these cells were found to drive the formation and growth of tumors $(3,8,12)$. Recent studies found that CSCs of human GBM can selfrenew and proliferate in TSM $(3,8)$. TSM known to be permissive for stem cell proliferation was established previously for isolation of neural stem cell as neurospheres $(3,8,20)$. Therefore, TSM become a common culture medium of human BTSCs.

Recently, we have isolated CSCs of human glioblastoma and identified the capacity of self-renewing, proliferation and tumor initiation. When we switched the human GBM primary culture cells into TSM containing EGF, bFGF and LIF, we also switched it into pure DMEM/F12 medium without exogenous growth factors or FBS. Neurosphere-like colonies appeared in all three GBM cultures after 1 week and reached $\sim 50$ cells each after 2 weeks in pure DMEM/F12 medium. This raises the question: do the tumor spheres consist of BTSCs? To answer this question, the sphere cells were subjected to a selfrenewing, proliferation and initiating tumor assay.

In this study, we have identified that the tumor sphere cells cultured in pure DMEM/F12 medium consist of BTSCs. There are several lines of evidence that support this: a) the tumor sphere cells showed immunoreactivity for CD133, a neural stem cell marker and BTSC marker; (21-25) b) the tumor sphere cells can self-renew and proliferate to generate subspheres that express CD133; c) the isolated tumor sphere cells can produce brain tumors in nude mice, its capacity of initiating tumor is much stronger than the cultured primary glioblastoma cells. Then, it is intriguing to speculate why BTSCs could self-renew and proliferate in pure DMEM/F12 medium. Are the self-renewal and proliferation of BTSCs independent of exogenous growth factors?

The serum-free medium (SFM) of culturing BTSCs used previously contained bFGF, EGF, LIF and/or PDGF. SFM allows for the maintenance of an undifferentiated stem cell state, and the addition of bFGF and EGF in TSM induced the proliferation of multipotent, self-renewing, and expandable neural stem cells $(11,26,27)$. Our data show that the BTSCs of human glioblastoma could self-renew and proliferate in pure DMEM/F12 medium without exogenous growth factors. What is the mechanism? Previously, GBM cells were shown to expressed EGFR, FGFR and PDGFR and they could secrete several autocrine factors such as EGF, bFGF, PDGF and promoted invasion $(15,17,28-30)$. The autocrine factors can promote invasion of GBM cells and also can promote selfrenewing and proliferation of BTSCs. Results of RT-PCR and Western blot analysis show that there were expression of EGF and bFGF in three human GBM BTSCs. The data suggest that the autocrine factor may sustain BTSCs of human GBM self-renewal and proliferation.

In this study, we provide new insight into the self-renewal and proliferation of human BTSCs. With evidence of selfrenewal, proliferation and initiating tumor, we identified that
BTSCs can self-renew and proliferate in serum-free medium without exogenous growth factors. We also provide evidence to support that there were autocrine factors EGF and bFGF in human BTSCs. The finding of self-renewal in serum-free medium without exogenous growth factors and autocrine factor expression in BTSCs has important implications for understanding the molecular mechanisms of self-renewal and proliferation in human GBM BTSCs.

\section{References}

1. Lapidot T, Sirard C, Vormoor J, Murdoch B, Hoang T, Caceres-Cortes J, Minden M, Paterson B, Caligiuri M and Dick J: A cell initiating human acute myeloid leukaemia after transplantation into SCID mice. Nature 367: 645-648, 1994.

2. Al-Hajj M, Wicha M, Benito-Hernandez A, Morrison S and Clarke M: Prospective identification of tumorigenic breast cancer cells. Proc Natl Acad Sci USA 100: 3983-3988, 2003.

3. Singh SK, Clarke I, Terasaki M, Bonn V, Hawkins C, Squire J and Dirks P: Identification of a cancer stem cell in human brain tumors. Cancer Res 63: 5821-5828, 2003.

4. Prince ME and Ailles LE: Cancer stem cells in head and neck squamous cell cancer. J Clin Oncol 26: 2871-2875, 2008.

5. Sell S and Leffert HL: Liver cancer stem cells. J Clin Oncol 26: 2800-2805, 2008.

6. Soltysova A, Altanerova V and Altaner C: Cancer stem cells. Neoplasma 52: 435-440, 2005.

7. Reya T, Morrison SJ, Clarke MF and Weissman IL: Stem cells, cancer, and cancer stem cells. Nature 415: 105-111, 2001.

8. Yuan X, Curtin J, Xiong Y, Liu G, Waschsmann-Hogiu S, Farkas DL, Black KL and Yu JS: Isolation of cancer stem cells from adult glioblastoma multiforme. Oncogene 23: 9392-9400, 2004.

9. Singh SK, Clarke ID, Hide T and Dirks PB: Cancer stem cells in nervous system tumors. Oncogene 23: 7267-7273, 2004.

10. Li GH, Wang DL, Chen ZT, Yin CL, Ji H and Lv SQ: Isolation and identification of cancer stem cells in human gliomas. Chin J Cancer Prev Treat 14: 37-40, 2007.

11. Tropepe V, Sibilia M, Ciruna BG, Rossant J, Wagner EF and van der Kooy D: Distinct neural stem cells proliferate in response to EGF and FGF in the developing mouse telencephalon. Dev Biol 208: 166-188, 1999.

12. Kondo T, Setoguchi T and Taga T: Persistence of a small subpopulation of cancer stem-like cells in the C6 glioma cell line. Proc Natl Acad Sci USA 101: 781-786, 2004.

13. Welch WC, Kornblith PL, Michalopoulos GK, Petersen BE, Beedle A, Gollin SM and Goldfarb RH: Hepatocyte growth factor (HGF) and receptor (c-met) in normal and malignant astrocytic cells. Anticancer Res 19: 1635-1640, 1999.

14. Du Y and Dreyfus CF: Oligodendrocytes as providers of growth factors. J Neurosci Res 68: 647-564, 2002.

15. Hoelzinger DB, Demuth T and Berens ME: Autocrine factors that sustain glioma invasion and paracrine billogy in the brain microenvironment. J Natl Cancer Inst 99: 1583-1593, 2007.

16. Ramnarain DB, Park S, Lee DY, Hatanpaa KJ, Scoggin SO, Out H, Libermann TA, Raisanen JM, Ashfaq R, Wong ET, Wu J, Elliott R and Habib AA: Differential gene expression analysis reveals generation of an autocrine loop by a mutant epidermal growth factor receptor in glioma cells. Cancer Res 66: 867-874, 2006.

17. Kunkel P, Müller S, Schirmacher P, Stavrou D, Fillbrandt R, Westphal M and Lamszus K: Expression and localization of scatter factor/hepatocyte growth factor in human astrocytomas. Neurooncol 3: 82-88, 2001.

18. Al-Hajj M, Becker MW, Wicha M, Weissman I and Clarke MF: Therapeutic implications of cancer stem cells. Curr Opin Genet Dev 14: 43-47, 2004.

19. Oliver TG and Wechsler-Reya RJ: Getting at the root and stem of brain tumors. Neuron 42: 885-888, 2004.

20. Reynolds BA and Weiss S: Generation of neurons and astrocytes from isolated cells of the adult mammalian central nervous system. Science 255: 1707-1710, 1992.

21. Yin AH, Miraglia S, Zanjani ED, Almeida-Porada G, Ogawa M, Leary AG, Olweus J, Kearney J and Buck DW: AC133, a novel marker for human hematopoietic stem and progenitor cells. Blood 90: 5002-5012, 1997. 
22. Uchida N, Buck DW, He D, Reitsma MJ, Masek M, Phan TV, Tsukamoto AS, Gage FH and Weissman IL: Direct isolation of human central nervous system stem cells. Proc Natl Acad Sci USA 97: 14720-14725, 2000.

23. Miraglia S, Godfrey W, Yin AH, Atkins K, Warnke R, Holden JT, Bray RA, Waller EK and Buck DW: A novel five-transmembrane hematopoietic stem cell antigen: isolation, characterization, and molecular cloning. Blood 90: 5013-5021, 1997.

24. Bidlingmaier S, Zhu X and Liu B: The utility and limitations of glycosylated human CD133 epitopes in defining cancer stem cells. J Mol Med 86: 1025-1032, 2008.

25. Mizrak D, Brittan M and Alison MR: CD133: molecule of the moment. J Pathol 214: 3-9, 2008.

26. Reynolds BA and Weiss S: Clonal and population analyses demonstrate that an EGF-responsive mammalian embryonic CNS precursor is a stem cell. Dev Biol 175: 1-13, 1996.

27. Reynolds BA, Tetzlaff W and Weiss S: A multipotent EGFresponsive striatal embryonic progenitor cell produces neurons and astrocytes. J Neurosci 12: 4565-4574, 1992.
28. Steiner HH, Karcher S, Mueller MM, Nalbantis E, Kunze S and Herold-Mende C: Autocrine pathways of the vascular endothelial growth factor (VEGF) in glioblastoma multiforme: clinical relevance of radiation-induced increase of VEGF levels. J Neurooncol 66: 129-138, 2004.

29. Allerstorfer S, Sonvilla G, Fischer H, Spiegl-Kreinecker S, Gauglhofer C, Setinek U, Czech T, Marosi C, Buchroithner J, Pichler J, Silye R, Mohr T, Holzmann K, Grasl-Kraupp B, Marian B, Grusch M, Fischer J, Micksche M and Berger W: FGF5 as an oncogenic factor in human glioblastoma multiforme: autocrine and paracrine activities. Oncogene 27: 4180-4190, 2008.

30. Ma D, Nutt CL, Shanehsaz P, Peng X, Louis DN and Kaetzel DM: Autocrine platelet-derived growth factor-dependent gene expression in glioblastoma cells is mediated largely by activation of the transcription factor sterol regulatory element binding protein and is associated with altered genotype and patient survival in human brain tumors. Cancer Res 65: 5523-5534, 2005. 